OPEN ACCESS

Edited by:

Dawei Wu,

Massachusetts Eye \& Ear Infirmary and Harvard Medical School,

United States

Reviewed by:

Michelle Menon Miyake,

Clinica Menon, Brazil Olga Krysko,

Ghent University, Belgium

${ }^{*}$ Correspondence:

Sarina K. Mueller

sarina.mueller@uk-erlangen.de

Specialty section:

This article was submitted to

Clinical Microbiology,

a section of the journal

Frontiers in Cellular and

Infection Microbiology

Received: 10 November 2021

Accepted: 20 December 2021

Published: 28 January 2022

Citation:

Mueller SK (2022) The Role of Exosomes in the Pathophysiology of

Chronic Rhinosinusitis.

Front. Cell. Infect. Microbiol. 11:812920.

doi: 10.3389/fcimb.2021.812920

\section{The Role of Exosomes in the Pathophysiology of Chronic Rhinosinusitis}

\author{
Sarina K. Mueller* \\ Department of Otolaryngology, Head and Neck Surgery, Friedrich-Alexander-Universität Erlangen-Nürnberg (FAU), \\ Erlangen, Germany
}

Non-invasive biomarker analysis has made repetitive and painless sampling over time possible. Exosomes are being released from a parent cell and their cargo mirrors the cell micromilieu of the parent cell. Therefore, exosomes are promising surrogates for their parent cells. That is also why exosomes provide an improved signal-to-noise ratio. Current studies have identified valid non-invasive biomarkers that may be able to monitor disease severity. Exosomes are suggested to play an important role in interepithelial communication and are suggested to play a role in the initiation and maintenance of inflammation in CRS. They are, however, also involved simultaneously in several immunological processes including immune protection and immunosuppression. As the isolation of exosomes is time-consuming their value in everyday routine diagnostics has yet to be determined.

Keywords: chronic rhinosinusitis (CRS), exosome, cystatin, pappalysin-1, cell communication

\section{INTRODUCTION}

Chronic rhinosinusitis (CRS) is one of the most common diseases worldwide and leads to a high socioeconomical burden. The annual direct costs (medication, surgery) or indirect costs (days off, reduced labor productivity) add up to 20 billion US\$ (USA 2017) (Rudmik, 2017). CRS patients report the subjective reduction in their quality of life as being worse than patients with chronic heart failure or chronic obstructive pulmonary disease (COPD) (Metson and Gliklich, 2000; Soler et al., 2011). Diagnosis is based on the ICAR : RS (Orlandi et al., 2021) criteria. The existence of a type 2 response, including tissue/serum eosinophils, tissue/serum IgE and the cytokines Il-4, Il-5 and Il-13, is currently the only measure for classifying the disease and predicting potential therapies (Fokkens et al., 2020). The identification and validation of specific and sensitive biomarkers that can diagnose different subtypes of the disease and predict its course are a main priority. Biomarkers can be analyzed in tissue, blood, and nasal secretions (Maxfield et al., 2018; Khan et al., 2019; Mueller et al., 2019a). Tissue biomarkers reflect local nasal inflammatory processes and the nasal microenvironment most closely. However, tissue biopsies from sinus mucosa can only be sampled invasively and at the time point of surgery. Presurgical or longitudinal sampling is not possible. This is why non-invasive mediums, including peripheral blood and nasal secretions, have been studied further. This means that painless and repetitive sampling over time and therefore 
longitudinal follow-up is possible (Massey et al., 2020). However, peripheral blood is a highly systemic medium representing pathophysiological processes of the whole body. Nasal mucus or nasal lavage fluid are easiest to sample and are accompanied by the lowest side effects. However, the respiratory mucosa is part of the first line of defense, and nasal secretions are therefore in contact with a multitude of pathogens. This is why the question of an improved signal-to-noise ratio for peripheral blood and nasal secretions arose. One answer for improving the signal-tonoise ratio is the use of exosomes (Mueller, 2018; Mueller et al., 2018; Mueller et al., 2019a). Exosomes are spherical to cupshaped, 30-150nm (Simons and Raposo, 2009; Raposo and Stoorvogel, 2013) vesicles which are secreted by virtually all cell types into a range of body fluids including nasal mucus (Simons and Raposo, 2009; Raposo and Stoorvogel, 2013). Exosomes incorporate proteins, lipids, DNA, miRNA and RNA that are specific to their cell of origin. Consequently, they can be used as a biomarker for their cell of origin (Pisitkun et al., 2006; Valadi et al., 2007; Qin and Xu, 2014). Due to their ubiquitous presence and easy accessibility, exosomes show great potential for non-invasive diagnostics and conducting "liquid biopsies" (Crowley et al., 2013).

Therefore, the objective of this review was to analyze the current literature concerning the role of exosomes in the pathophysiology of CRS.

\section{ADVANTAGES AND DISADVANTAGES IN THE USAGE OF EXOSOMES}

Exosomes are cell specific and reflect cell functions and conditions. This is why they show great potential as novel biomarkers for clinical diagnosis. Furthermore, exosomes protect their cargo from degradation by nucleases and proteases, thereby increasing the biomarker half-life. Exosomes resemble the tissue proteome more closely than whole mucus and show an improved signal-to-noise ratio (Mueller et al., 2019a). However, the handling of exosomes is not trivial. The majority of current techniques of exosomes processing only concentrates the exosomes and does not isolate them. Consequently, there may be chance of incorrect conclusions regarding biological activity and function of exosomes (Mueller et al., 2018). The most common technique for exosome concentration is ultracentrifugation procedure (Théry et al., 2006; Nocera et al., 2017). In a sequence of different centrifugation steps, exosomes may be successfully concentrated along with other particles with the same size and buoyant density. Ultracentrifugation is time consuming and requires costly equipment. Therefore, the application of exosomes in point-ofcare service may be difficult (Mueller et al., 2018). A technique that isolates the exosomes is based on immunoaffinity-based isolation. This technique uses antibody-coated magnetic beads and results in an improved output compared to ultracentrifugation. However, only exosomes containing the target protein can be isolated with that method (Tauro et al., 2012). Another frequently used method is the use of commercial precipitation kits. This method is probably the easiest to implement into clinical practice, however, the purity is reported to be lower as compared to ultracentrifugation techniques using a sucrose density gradient (Van Deun et al., 2014) Further isolation and concentration techniques are summarized in a specific review about exosomes in aerodigestive mucosa (Mueller et al., 2018).

\section{POTENTIAL NON-INVASIVE EXOSOMAL BIOMARKERS}

Several promising biomarkers have been analyzed in exosomes sampled from CRS patients. Those biomarkers include proteins (Mueller et al., 2018; Miyake MM et al., 2019; Mueller et al., 2019b; Mueller et al., 2019; Mueller et al., 2020; Shin et al., 2020) and miRNA (Shin et al., 2020; Zhang et al., 2020). Table 1 summarizes all exosomal biomarkers including their physiological function and their supposed role in CRS.

\section{Pathophysiological Roles of Exosomes in CRS} Role in Interepithelial Transfer

Intercellular communication is the transfer of cargo between an originating cell and a target cell. The traditional ways of intercellular communication are autocrine, paracrine and endocrine or by cell-to-cell-contacts (juxtacrine interaction) (Singh AB, 2005). However, a more sophisticated way of communication includes the exchange of information through the release of membrane-bound bodies called extracellular vesicles (Al-Nedawi et al., 2009). Extracellular vesicles including exosomes can transfer their cargo between close but also between distant cells by travelling through body fluids (Peinado et al., 2012). Therefore, the mechanism of transfer by extracellular vesicles may be interesting for various pathological states including the spreading of tumor cells or inflammatory markers (Peinado et al., 2012; Bleier et al., 2013). For CRS, exosomes may be sampled either from nasal mucus (Mueller et al., 2018; Miyake M et al., 2019b; Mueller et al., 2019c; Mueller et al., 2019; Mueller et al., 2020; Shin et al., 2020), nasal lavage fluid (Choi et al., 2014; Lässer et al., 2016; Zhang et al., 2020; Zhou et al., 2020; Wang et al., 2021) or tissue (Shenoy et al., 2020). In order to analyze the capability of exosomes to transfer cargo from cell to cell, in vitro and in vivo transfection experiments may be performed. In vitro exosomes may play a role in the initiation of inflammation after exposure with external stimuli e.g. air pollutants (Shin et al., 2020). Additionally, exosomes may play an even larger role in maintaining the inflammation in CRS by circling proinflammatory cargo from one cell to another (Bleier, 2012). Hereby, exosomes cannot only transfer cargo between autologous cells but also between epithelial cells of different anatomical regions (Zhang et al., 2020). Most importantly for the maintenance of inflammation, exosomes may transfer their cargo from a disease cell to a nondiseased (control). In the following, characteristic differentially expressed proteins measured to be upregulated in the diseased cell but not in the control cell, are found to also be overexpressed in the control cell after transfection (Zhou et al., 2020; Wang 
TABLE 1 | Summary of all exosomal biomarkers including their physiological function and their supposed role in CRS.

\begin{tabular}{|c|c|c|c|c|}
\hline Study & $\begin{array}{l}\text { Year } \\
\text { published }\end{array}$ & Biomarker & Physiologic function & role in CRS \\
\hline Lässer et al., 2016 & 2016 & Nitric oxide & $\begin{array}{l}\text { endothelium-derived relaxing factor (EDRF), e.g. } \\
\text { vasodilation }\end{array}$ & immune defense in respiratory tract \\
\hline Nocera et al., 2017 & 2017 & p-glycoprotein (P-gp) & ATP-binding cassette, anti-efflux membrane pump & immunomodulatory role in Th2 inflammation \\
\hline Mueller et al., 2018 & 2018 & serpins & $\begin{array}{l}\text { serine protease inhibitors, coagulation and fibrinolysis } \\
\text { cascade }\end{array}$ & coagulation and fibrinolysis cascade \\
\hline Mueller et al., 2019b & 2019 & serpins & $\begin{array}{l}\text { serine protease inhibitors, coagulation and fibrinolysis } \\
\text { cascade }\end{array}$ & coagulation and fibrinolysis cascade \\
\hline Nocera et al., 2019 & 2019 & Nitric oxide & $\begin{array}{l}\text { endothelium-derived relaxing factor (EDRF), e.g. } \\
\text { vasodilation }\end{array}$ & $\begin{array}{l}\text { immune defense, innate } \\
\text { immunosurveillance }\end{array}$ \\
\hline Miyake MM et al., 2019 & 2019 & cystatin-SA (CST-2) & cysteine protease inhibitor & $\begin{array}{l}\text { segregation of phenotypes, disease severity } \\
\text { monitoring }\end{array}$ \\
\hline Zhang et al., 2020 & 2020 & miRNA-22 (MiR-22) & microRNA, mediation of inhibition of translation & enhancement of tubular permeability \\
\hline Shin et al., 2020 & 2020 & miRNA-19A/miRNA-61 & microRNA, mediation of inhibition of translation & $\begin{array}{l}\text { promotion of proinflammatory macrophage } \\
\text { polarization }\end{array}$ \\
\hline Mueller et al., 2020 & 2020 & pappalysin-1 (PAPP-A) & $\begin{array}{l}\text { metalloendopeptidase/secreted protease, growth } \\
\text { promotion }\end{array}$ & $\begin{array}{l}\text { early recurrence prediciton, disease severity } \\
\text { monitoring }\end{array}$ \\
\hline Wang et al., 2021 & 2021 & MUC-5A & glycoprotein, first line of defense in respiratory tract & induction of proinflammatory proteins \\
\hline
\end{tabular}

et al., 2021). It is suspected that the interepithelial transfer is similar in vivo (Nocera et al., 2019).

\section{Role in Immune Response}

For CRS, several endotypes have been described for CRSwNP and CRSsNP (Tomassen et al., 2016). Comparable to immune responses in the lower airways, Th1- and Th2- responses have also been described for CRS. Originally, CRSwNP was associated with a Th2- based endotype whereas CRSsNP was associated with a Th1-response. However, the complexity of the immune response may be much greater than expected with different combinations of immune responses in one phenotype (Delemarre et al., 2020; Delemarre et al., 2021). It is suggested that mRNAs or proteins that play a role in inflammatory immune mechanisms may be transferred by exosomes (Wang et al., 2021). Therefore, inflammation may be spread between epithelial cells. Simultaneously, exosomes may also contain information for fighting inflammatory processes and carry immune protective content (Lässer et al., 2016; Nocera et al., 2019). In this context, exosomes may attract immune cells of pathological epithelial cells from CRS or asthma patients. A migration of monocytes, neutrophils and NK cells have been described in vitro (Lässer et al., 2016). When analyzing the exosomal proteome, it could be strongly correlated with proas well as anti-inflammatory proteins which may be present at the same time (Lässer et al., 2016; Mueller et al., 2019a). One enzyme that was found to be biologically active even within exosomes was the inducible nitric oxide synthase (iNOS) (Lässer et al., 2016; Nocera et al., 2019). Nitric oxide may play an important role in the immune protection of the airway epithelium as a first line of defense. NO can also be induced by lipopolysaccharides (LPS). LPS can be found in the outer membrane of Gram-negative bacteria and are known to stimulate toll-like receptor 4 (TLR-4). It could be shown that LPS lead to a secretion of exosomes into nasal mucus in vitro and in vivo (Nocera et al., 2019). Those exosomes increased in number after LPS induction and exosomal inducible nitric ocide synthase expression was also increased. It is suggested that the LPS stimulation led to increased exosomal microbiocidal activity against Pseudomonas aeruginosa. Moreover, NO may be transferred between epithelial cells, thus increasing NO production within nasal epithelial cells (Nocera et al., 2019). Interestingly, transfection experiments indicate that the protein transfer was completed within 5 minutes of contact (Nocera et al., 2019). Hence, exosomes may therefore play a role in the innate immunosurveillance and defense mechanism of the human upper airways. Nasal mucus derived exosomes seem to harbor immune defensive proteins, which suggests that exosomes may also play a role in maintaining the barrier function of the sinonasal mucosa.

\section{Role in Predicting Disease Severity and Recurrence}

Despite CRS being a common disease, the pathophysiology of recurrences as well as the prediction of disease severity remain elusive. Nor has a common definition of recurrence has been established. A classification based on the time point of recurrence or disease severity based on endotypes is desperately needed. Exosomal research on protein biomarkers provides one approach to solving this shortcoming. Nasal mucus derived exosomes may contain cystatin-SA (CST-2), a cysteine protease inhibitor. Exosomal CST-2 was correlated with clinical and demographical variables including allergies, asthma, eosinophilia and nonsteroidal anti-inflammatory respiratory disease-exacerbated respiratory disease. It is suggested that CST-2 expression can segregate the phenotype. Additionally, CST-2 levels may cluster CRS patients based on disease severity (Miyake MM et al., 2019). CST-2 may moreover play a part in disease recurrence as do periostin and pappalysin-1. Although the study was performed in whole mucus all protein biomarkers have been shown to also be exosomal (Mueller et al., 2019a; Mueller et al., 2020). All biomarkers decreased after sinus surgery and increased postoperatively. An earlier and steeper increase correlated with 
earlier recurrences. An increase in the combination of periostin, CST-2 and pappalysin-1 may therefore predict early recurrences in CRS. Interestingly, this combination of biomarkers increased their levels months before the patients experienced symptoms measured with the rhinologic domain of the SNOT-22 score (Mueller et al., 2019c). Those nasal mucus derived proteins may therefore play a role in the diagnosis of exacerbations and the prediction of recurrences.

\section{Role in the Coagulation and Fibrinolysis Pathways}

The coagulation and fibrinolysis pathways have been studied by several groups with respect to CRS (Takabayashi et al., 2013; Kim et al., 2015). Among all protein pathways analyzed by a multiplexed approach, the coagulation cascade was the most significantly associated with CRSwNP (Mueller et al., 2019b). It is suggested that the coagulation cascade is upregulated whereas the fibrinolysis cascade is downregulated. As a consequence, fibrin accumulates which leads to increased edema and polyp growth. These findings could not only been noted in CRSwNP tissue but also in nasal mucus-derived exosomes from CRSwNP patients (Mueller et al., 2018; Mueller et al., 2019b). As the coagulation and fibrinolysis cascades are complex due to their frequent interaction with other pathways, sufficient substrate is necessary for analysis. Due to the non-invasive and repetitive sampling possibilities, exosomes represent one method that allows frequent sampling of high-quality substrate.

\section{Role in Microbiome}

"The microbiome is the collection of all microbes, such as bacteria, fungi, viruses and their genes that live naturally on our bodies and inside us" (https://www.niehs.nih.gov/). Regarding external causes to explain the etiology of CRS, different bacteria have been discussed. Interestingly, exosomes may also be released by bacteria and therefore may be an important causative agent of inflammation. The metagenomic analysis of the nasal lavage fluid microbiome showed that the composition of the bacteria was positively correlated with the exosome composition. For bacteria and their exosomes, patients with CRS had greater bacterial abundance and lower diversity compared to controls. Additionally, an increased Staphylococcus aureus abundance was seen in CRSwNP patients (Choi et al., 2014). This mechanism may be one explanation for the etiology of CRS and the initiation of inflammation by exosomal cargo from bacteria.

\section{Role Immune Suppression by T Cell Checkpoints}

Immune checkpoints are physiologically integrated into the immune system. Their role is to prevent an excessive immune response that destroys healthy cells in the body. At the latest since the Nobel Prize was presented to Tasuku Honjo and James P. Allison in 2018, immune checkpoint inhibitors, including programmed cell death protein-1 (PD-1) or cytotoxic Tlymphocyte-associated Protein 4 (CTLA-4), have been ubiquitously present in the treatment of cancer. Accordingly, research on $\mathrm{T}$ cells and their activation patterns has also been carried out in CRSwNP. As already mentioned in previous paragraphs, exosomes may contain immunogenic as well as immunosuppressive content. In this context, tissue exosomes of CRSwNP patients may be able to arrest the activation of T cells stimulated by the $\mathrm{T}$ cell receptor. Similarly to the tumor microenvironment, these exosomes can lead to an immune suppression and inhibit $\mathrm{T}$ cell activation. In CRSwNP, this tumor suppression may be dependent on ganglioside GD3, which is expressed on the exosomal surface. Consequently, exosomes with immunosuppressive characteristics may represent a $\mathrm{T}$ cell checkpoint and therefore a target for novel treatment options (Shenoy et al., 2020).

\section{SHORTCOMINGS AND FUTURE PERSPECTIVES}

Exosomal research is a small but increasing field of activity in rhinology and in studying CRS. The non-invasive sampling through whole nasal mucus or nasal lavage fluid has made painless and repetitive sampling over time possible. The usage of exosomes is beneficial due to the improved signal-to-noiseratio. Although the application of exosomal biomarkers would be suitable, today, no study has analyzed the potential for the diagnosis or prediction of acute exacerbations. Ideally, an exosomal biomarker would increase before subjective symptoms of an acute exacerbation of CRS (AECRS) emerge. This would give the physician the time to react and if necessary, escalate medical therapy. Future studies in this area are urgently needed. Importantly, a unified definition of AECRS should be used in those studies to guarantee comparability and uniformity. Promising biomarkers of the current literature may be evaluated specifically for AECRS (Mueller et al., 2019c; Wu et al., 2019; Wu et al., 2020).

Exosomes may represent one approach to explain interepithelial communication. However, it is not entirely clear which cargo ultimately ends up in the exosome to be transported to neighboring cells. Additionally, it is not clear how many proteins or mRNAs are needed to be differentially expressed in order to lead to a pathological change in the receiving cell. These questions would be crucial to answer as the transfer of pathological proteins comprises the chance of interfering with novel therapeutic options. Future studies are needed to solve these shortcomings.

\section{CONCLUSIONS}

Various exosomal biomarkers have been investigated. Exosomal biomarkers may play an important role in the prediction of the severity of CRS, polyp growth and recurrence, the initiation and maintenance of inflammation, vascular permeability and immune protection as well as immunoinhibition. Several studies have suggested that exosomes play a crucial role in the transport of their cargo from one cell to another, even between different epithelial cell origins. This mechanism may be a key for spreading and maintaining disease and is one step towards the analysis of 
the pathophysiology of CRS. Research on exosomes allows studying the pathophysiology of CRS as well as prospective biomarkers. However, in this relatively new field, future studies are needed to validate the results.

\section{REFERENCES}

Available at: https://www.niehs.nih.gov/.

Al-Nedawi, K., Meehan, B., and Rak, J. (2009). Microvesicles: Messengers and Mediators of Tumor Progression. Cell Cycle 8 (13), 2014-2018. doi: 10.4161/ cc.8.13.8988

Bleier, B. (2012). Regional Expression of Epithelial MDR1/P-Glycoprotein in Chronic Rhinosinusitis With and Without Nasal Polyposis. Int. Forum Allergy Rhinol. 2, 122-125. doi: 10.1002/alr.21004

Bleier, B., Nocera, A., Iqbal, H., Hoang, J., Feldman, R., and Han, X. (2013). PGlycoprotein Functions as an Immunomodulator in Healthy Human Primary Nasal Epithelial Cells. Int. Forum Allergy Rhinol. 3 (6), 433-438. doi: 10.1002/ alr.21166

Choi, E. B., Hong, S. W., Kim, D. K., Jeon, S. G., Kim, K. R., Cho, S. H., et al. (2014). Decreased Diversity of Nasal Microbiota and Their Secreted Extracellular Vesicles in Patients With Chronic Rhinosinusitis Based on a Metagenomic Analysis. Allergy 69 (4), 517-526. doi: 10.1111/all.12374

Crowley, E., Di Nicolantonio, F., Loupakis, F., and Bardelli, A. (2013). Liquid Biopsy: Monitoring Cancer-Genetics in the Blood. Nat. Rev. Clin. Oncol. 10 (8), 472-484. doi: 10.1038/nrclinonc.2013.110

Delemarre, T., Holtappels, G., De Ruyck, N., Zhang, N., Nauwynck, N., Bachert, H., et al (2020). Type 2 Inflammation in Chronic Rhinosinusitis Without Nasal Polyps: Another Relevant Endotype. J. Allergy Clin. Immunol. 146 (2), 337343.36. doi: 10.1016/j.jaci.2020.04.040

Delemarre, T., Holtappels, G., De Ruyck, N., Zhang, N., Nauwynck, N., Bachert, H., et al. (2021). A Substantial Neutrophilic Inflammation as Regular Part of Severe Type 2 Chronic Rhinosinusitis With Nasal Polyps. J. Allergy Clin. Immunol. 147 (1), 179-188.e2. doi: 10.1016/j.jaci.2020.08.036

Fokkens, W. J., Lund, V. J., Hopkins, C., Hellings, P. W., Kern, R., Reitsma, S., et al. (2020). European Position Paper on Rhinosinusitis and Nasal Polyps 2020. Rhinology 58 (Suppl S29), 1-464. doi: 10.4193/Rhin20.600

Khan, A., Huynh, T., Vandeplas, G., Joish, V. N., Mannent, L. P., Tomassen, P., et al. (2019). The GALEN Rhinosinusitis Cohort: Chronic Rhinosinusitis With Nasal Polyps Affects Health-Related Quality of Life. Rhinology 57 (5), 343-351. doi: 10.4193/Rhin 19.158

Kim, D., Cho, S., Takabayashi, T., and Schleimer, R. (2015). Chronic Rhinosinusitis and the Coagulation System. Allergy Asthma Immunol. Res. 7 (5), 421-430. doi: 10.4168/aair.2015.7.5.421

Lässer, C., O’Neil, S. E., Shelke, G. V., Sihlbom, C., Hansson, S. F., Gho, Y. S., et al. (2016). Exosomes in the Nose Induce Immune Cell Trafficking and Harbour an Altered Protein Cargo in Chronic Airway Inflammation. J. Transl. Med. 14 (1), 181. doi: 10.1186/s12967-016-0927-4

Massey, C., Diaz Del Valle, F., Abuzeid, W., Levy, J. M., Mueller, S., Levine, C. G., et al. (2020). Sample Collection for Laboratory-Based Study of the Nasal Airway and Sinuses: A Research Compendium. Int. Forum Allergy Rhinol. 2. 10 (3), 303-313. doi: 10.1002/alr.22510

Maxfield, A., Landegger, L., and Brook, C. (2018). Periostin as a Biomarker for Nasal Polyps in Chronic Rhinosinusitis. Otolaryngol. Head Neck Surg. 158 (1), 181-186. doi: 10.1177/0194599817737967

Metson, R., and Gliklich, R. (2000). Clinical Outcomes in Patients With Chronic Sinusitis. Laryngoscope 110, 24-28. doi: 10.1097/00005537-200003002-00007

Miyake, M. M., Workman, A. D., Nocera, A. L., Wu, D., Mueller, S. K., Finn, K., et al. (2019). Discriminant Analysis Followed by Unsupervised Cluster Analysis Including Exosomal Cystatins Predict Presence of Chronic Rhinosinusitis, Phenotype, and Disease Severity. Int. Forum Allergy Rhinol. 9 (9), 1069-1076. doi: 10.1002/alr.22380

Miyake, M. M., Workman, A. D., Nocera, A. L., Wu, D., Mueller, S. K., Finn, K., et al. (2019). Exosomal Cystatins Predict Chronic Rhinosinusitis, Phenotype, and Disease Severity. Int. Forum Allergy Rhinol. 9 (9), 1069-1076. doi: 10.1002/ alr. 22380

\section{AUTHOR CONTRIBUTIONS}

The author confirms being the sole contributor of this work and has approved it for publication.

Mueller, S. K. (2018). Exosomes and Chronic Rhinosinusitis. World J Otorhinolaryngol. - Head Neck Surg. 4 (3), 175-178. doi: 10.1016/ j.wjorl.2018.07.005

Mueller, S. K., Nocera, A. L., and Bleier, B. S. (2018). Exosome Function in Aerodigestive Mucosa. Nanomed. Nanotechnol. Biol. Med. 14 (2), 269-277. doi: 10.1016/j.nano.2017.10.008

Mueller, S., Nocera, A., Dillon, S., Gu, X., Wendler, O., Otu, H. H., et al. (2019a). Non-Invasive Exosomal Proteomic Biosignatures Including Cystatin-SN (CST 1), Peroxiredoxin-5 (PRDX5) and Glycoprotein-VI (GP6) Accurately Predict Chronic Rhinosinusitis With Nasal Polyps. Int. Forum Allergy Rhinol. 9 (2), 177-186. doi: 10.1002/alr.22226

Mueller, S., Nocera, A., Dillon, S., Libermann, T., Wendler, O., and Bleier, B. (2019b). Tissue and Exosomal Serine Protease Inhibitors Are Significantly Overexpressed in Chronic Rhinosinusitis With Nasal Polyps. Am. J. Rhinol. Allergy 27, 1945892. doi: 10.1177/1945892419831108

Mueller, S. K., Nocera, A. L., Dillon, S. T., Wu, D., Libermann, T. A., and Bleier, B. S. (2018). Highly Multiplexed Proteomic Analysis Reveals Significant Tissue and Exosomal Coagulation Pathway Derangement in Chronic Rhinosinusitis With Nasal Polyps. Int. Forum Allergy Rhinol. 8 (12), 1438-1444. doi: 10.1002/ alr.22189

Mueller, S. K., Nocera, A. L., Workman, A., Libermann, T., Dillon, S. T., Stegmann, A., et al. (2020). Significant Polyomic and Functional Upregulation of the PAPP-A/ IGFBP-4/5/IGF-1 Axis in Chronic Rhinosinusitis With Nasal Polyps. Int. Forum Allergy Rhinol. 10 (4), 546-555. doi: 10.1002/alr.22512

Mueller, S., Wendler, O., Nocera, A., Grundtner, P., Schlegel, P., Agaimy, A., et al. (2019). Escalation in Mucus Cystatin 2, Pappalysin-A and Periostin Levels Over Time Predict Need for Recurrent Surgery in CRSwNP. Int. Forum Allergy Rhinol. 9 (10), 1212-1219. doi: 10.1002/alr.22407

Nocera, A., Miyake, M., Seifert, P., Han, X., and Bleier, B. (2017). Exosomes Mediate Inter-Epithelial Transfer of Functional P-Glycoprotein in Chronic Rhinosinusitis With Nasal Polyps. Laryngoscope 127 (9), E295-E300. doi: 10.1002/lary.26614

Nocera, A. L., Mueller, S. K., Stephan, J. R., Hing, L., Seifert, P., Han, X., et al. (2019). Exosome Swarms Eliminate Airway Pathogens and Provide Passive Epithelial Immunoprotection Through Nitric Oxide. J. Allergy Clin. Immunol. 143 (4), 1525-1535.e1. doi: 10.1016/j.jaci.2018.08.046

Orlandi, R. R., Kingdom, T. T., Smith, T. L., Bleier, B., DeConde, A, Luong, A. U., et al. (2021). International Consensus Statement on Allergy and Rhinology: Rhinosinusitis 2021. Int. Forum Allergy Rhinol. 11 (3), 213-739. doi: 10.1002/alr.22741

Peinado, H., Aleckovic, M., Lavotshkin, S., Matei, I., Costa-Silva, B., MorenoBueno, G., et al (2012). Melanoma Exosomes Educate Bone Marrow Progenitor Cells Toward a Pro-Metastatic Phenotype Through MET. Nat. Med. 18, $883-$ 891. doi: $10.1038 / \mathrm{nm} .2753$

Pisitkun, T., Johnstone, R., and Knepper, M. A. (2006). Discovery of Urinary Biomarkers. Mol. Cell Proteomics 5 (10), 1760-1771. doi: 10.1074/mcp.R600004MCP200

Qin, J., and Xu, Q. (2014). Functions and Applications of Exosomes. Acta Pol. Pharm. 71, 537-543.

Raposo, G., and Stoorvogel, W. (2013). Extracellular Vesicles: Exosomes, Microvesicles, and Friends. J. Cell Biol. 200 (4), 373-383. doi: 10.1083/ jcb. 201211138

Rudmik, L. (2017). Economics of Chronic Rhinosinusitis. Curr. Allergy Asthma Rep. 17, 20. doi: 10.1007/s11882-017-0690-5

Shenoy, G., Bhatta, M., Loyall, J., Kelleher, R. J., Bernstein, J., and Bankert, R. (2020). Exosomes Represent an Immune Suppressive T Cell Checkpoint in Human Chronic Inflammatory Microenvironments. Immunol. Invest. 49 (7), 726-743. doi: 10.1080/08820139.2020.1748047

Shin, C., Byun, J., Lee, K., Kim, B., Noh, Y. K., T, N. L., et al. (2020). Exosomal miRNA19a and miRNA-614 Induced by Air Pollutants Promote Proinflammatory M1 Macrophage Polarization via Regulation of RORalpha Expression in Human 
Respiratory Mucosal Microenvironment. J. Immunol. 205 (11), 3179-3190. doi: 10.4049/jimmunol.2000456

Simons, M., and Raposo, G. (2009). Exosomes-Vesicular Carriers for Intercellular Communication. Curr. Opin. Cell Biol. 21, 575-581. doi: 10.1016/j.ceb.2009.03.007

Singh AB, H. R. (2005). Autocrine, Paracrine and Juxtacrine Signaling by EGFR Ligands. Cell Signal. 17, 1138-1193. doi: 10.1016/j.cellsig.2005.03.026

Soler, Z., Wittenberg, E., Schlosser, R., Mace, J., and Smith, T. (2011). Health State Utility Values in Patients Undergoing Endoscopic Sinus Surgery. Laryngoscope 121 (12), 2672-2678. doi: 10.1002/lary.21847

Takabayashi, T., Kato, A., Peters, A., Hulse, K. E., Suh, L. A., Carter, R., et al. (2013). Increased Expression of Factor XIII-A in Patients With Chronic Rhinosinusitis With Nasal Polyps. J. Allergy Clin. Immunol. 132 (3), 584592. doi: 10.1016/j.jaci.2013.02.003

Tauro, B., Greening, D., Rommel, A., Ji, H., Mathivanan, S., Scott, A. M., et al. (2012). Comparison of Ultracentrifugation, Density Gradient Separation, and Immunoaffinity Capture Methods for Isolating Human Colon Cancer Cell Line LIM1863-Derived Exosomes. Methods 56 (2), 293-304. doi: 10.1016/ j.ymeth.2012.01.002

Théry, C., Amigorena, S., Raposo, G., and Clayton, A. (2006). Isolation and Characterization of Exosomes From Cell Culture Supernatants and Biological Fluids. Curr. Protoc. Cell Biol 3, Unit 3.22. doi: 10.1002/0471143030.cb0322s30

Tomassen, P., Vandeplas, G., Van Zele, T., Cardell, L., and Al, E. (2016). Inflammatory Endotypes of Chronic Rhinosinusitis Based on Cluster Analysis of Biomarkers. J. Allergy Clin. Immunol. V. 137 (5), 1449-1456.e4. doi: 10.1016/j.jaci.2015.12.1324

Valadi, H., Ekström, K., Bossios, A., Sjöstrand, M., Lee, J., and Lötvall, J. (2007). Exosome-Mediated Transfer of mRNAs and microRNAs is a Novel Mechanism of Genetic Exchange Between Cells. Nat. Cell Biol. 9 (6), 654656. doi: $10.1038 /$ ncb1596

Van Deun, J., Mestdagh, P., Sormunen, R., Cocquyt, V., Vermaelen, K., Vandesompele, J., et al. (2014). The Impact of Disparate Isolation Methods for Extracellular Vesicles on Downstream RNA Profiling. J. Extracell. Vesicles 3, 1-14. doi: 10.3402/jev.v3.24858

Wang, L., Lee, C., Liang, S., Hung, C. C., Wu, Y. R., Chien, C. Y., et al. (2021). Mucin 5AC Is Significantly Upregulated in Exosomes From the Nasal Lavage
Fluid and may Promote the Expression of COX-2, VEGF and MMP-9: An Implication in Nasal Polyp Pathogenesis. Rhinology 59 (3), 328-336. doi: 10.4193/Rhin 20.564

Wu, D., Bleier, B., and Wei, Y. (2019). Current Understanding of the Acute Exacerbation of Chronic Rhinosinusitis. Front. Cell Infect. Microbiol. 9, 415. doi: $10.3389 /$ fcimb.2019.00415

Wu, D., Bleier, B., and Wei, Y. (2020). Definition and Characteristics of Acute Exacerbation in Adult Patients With Chronic Rhinosinusitis: A Systematic Review. J. Otolaryngol. Head Neck Surg. 49 (1), 62. doi: 10.1186/s40463-02000459-w

Zhang, W., Zhang, T., Yan, Y., Zhang, J., Zhou, Y., Pei, Y., et al. (2020). Exosomal miR-22-3p Derived From Chronic Rhinosinusitis With Nasal Polyps Regulates Vascular Permeability by Targeting VE-Cadherin. BioMed. Res. Int. 1237678. doi: $10.1155 / 2020 / 1237678$

Zhou, M., Sen, T. K., Guan, W. J., Jian, L. J., Deng, J., Gao, W. X., et al. (2020). Proteomics Profiling of Epithelium-Derived Exosomes From Nasal Polyps Revealed Signaling Functions Affecting Cellular Proliferation. Respir. Med. 162 (58):105871. doi: 10.1016/j.rmed.2020.105871

Conflict of Interest: The author declares that the research was conducted in the absence of any commercial or financial relationships that could be construed as a potential conflict of interest.

Publisher's Note: All claims expressed in this article are solely those of the authors and do not necessarily represent those of their affiliated organizations, or those of the publisher, the editors and the reviewers. Any product that may be evaluated in this article, or claim that may be made by its manufacturer, is not guaranteed or endorsed by the publisher.

Copyright $\odot 2022$ Mueller. This is an open-access article distributed under the terms of the Creative Commons Attribution License (CC BY). The use, distribution or reproduction in other forums is permitted, provided the original author(s) and the copyright owner(s) are credited and that the original publication in this journal is cited, in accordance with accepted academic practice. No use, distribution or reproduction is permitted which does not comply with these terms. 